\title{
Checkrein deformity after ankle fracture
}

\begin{abstract}
Checkrein deformity may occur after leg or ankle fractures. It is a dynamic flexion deformity of the big toe after planter flextion of the ankle that disappear when the ankle is dorsiflexed. It is believed to be the result of Tethering or entrapment of the flexor hallucis longus (FHL) tendon in scar tissue or callus at the fracture site, ${ }^{1,2}$ It was also suggested that it results from contracture of the FHL muscles due to a subclinical or missed compartment syndrome. ${ }^{3}$ Checkrein deformity is usually noticed during follow up in clinic and it may not respond to conservative management like stretching exercises. Surgical treatment is best done at midfoot by FHL tendon lengthening over lengthening at the fracture site.
\end{abstract}

Keywords: checkrein deformity, FHL tendon, fracture site, dorsiflexed, hallux flexus

\author{
Volume II Issue 4 - 2019
}

Ahmad N Bo-Eisa, Mohammed S Alhassan
Department of Orthopedic, King Fahad Hospital, Saudi Arabia

Correspondence: Ahmad N Boeisa, Department of Orthopedic, King Fahad Hospital, Saudi Arabia, Email ahmadboeisa@gmail.com

Received: August 13,2019 | Published: August 27, 2019

\section{Background}

Flexor hallucis longus muscle can adhere to the distal tibia after tibial fracture. The patient may complain of deep posteromedial ankle pain, checkrein deformity of the hallux, hallux flexus or development of hallux rigidus. FHL tightness or checkrein deformity needs to be treated surgically either by lengthening or release of FHL muscle. ${ }^{1}$ Tethering of the flexor hallucis longus (FHL) tendon seen as a dynamic flexion deformity of the hallux. When the ankle is passively dorsiflexed, the flexion deformity becomes more prominent, but on passive plantar flexion the deformity is corrected completely. Checkrein deformity has been explained by either entrapment of the FHL in scar tissue or callus at the fracture site, ${ }^{2}$ but or as a results from contracture of the muscles after a subclinical compartment syndrome. ${ }^{3}$

\section{Case presentation}

Our case is 32 years old male presented to trauma pay after soccer
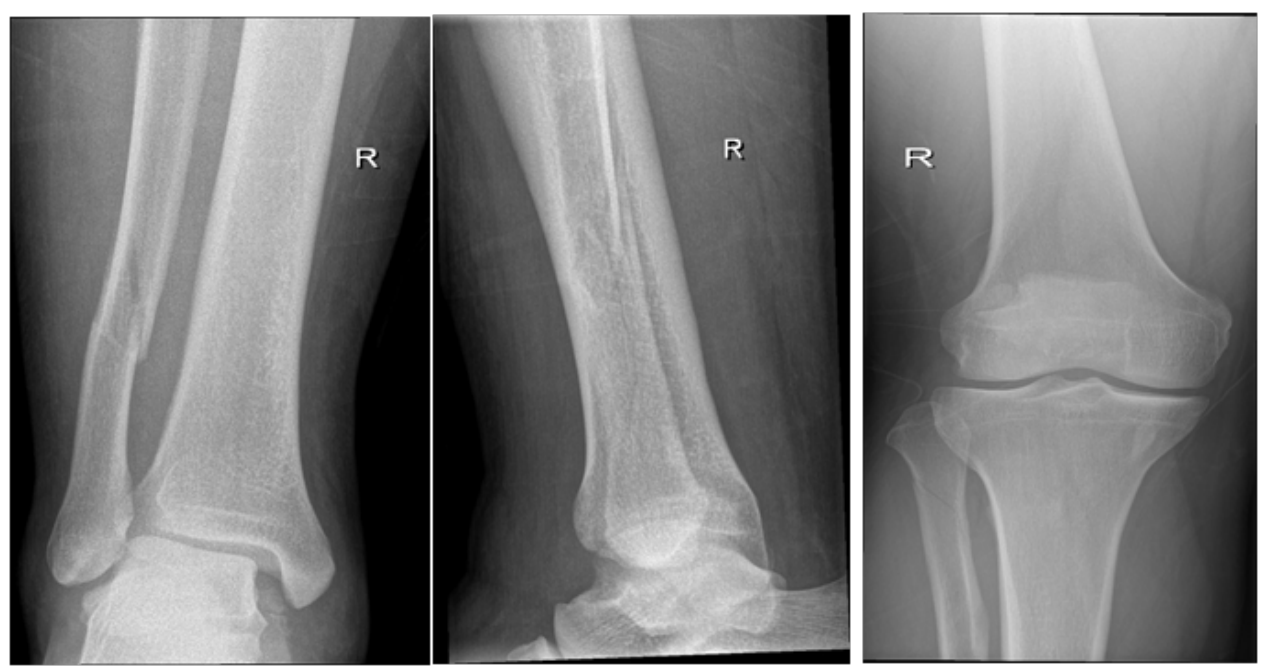

Figure I Primary right ankle X-rays done at trauma bay.

injury as he explained rotational mechanism. He presented with mild swelling and inability to bear weight. After investigation he was diagnosed with lateral malleolus fracture with failed syndesmotic ligament along with maisonneuve injury. Patient admitted to ward and next day he developed sever swelling and blister. Surgery delayed for soft tissue management about 1 week.

\section{Investigations}

Primary right Ankle X-rays done at trauma bay (Figure 1). AP and lateral X-rays of right ankle showing after fixation (Figure 2). After 8 weeks patient underwent synedsmotic screw removal (Figure 3), and he noticed big toe deformity. At 1 year follow up patient booked for implant removal and was still complaining of same deformity and underwent FHL lengthening at same time. 


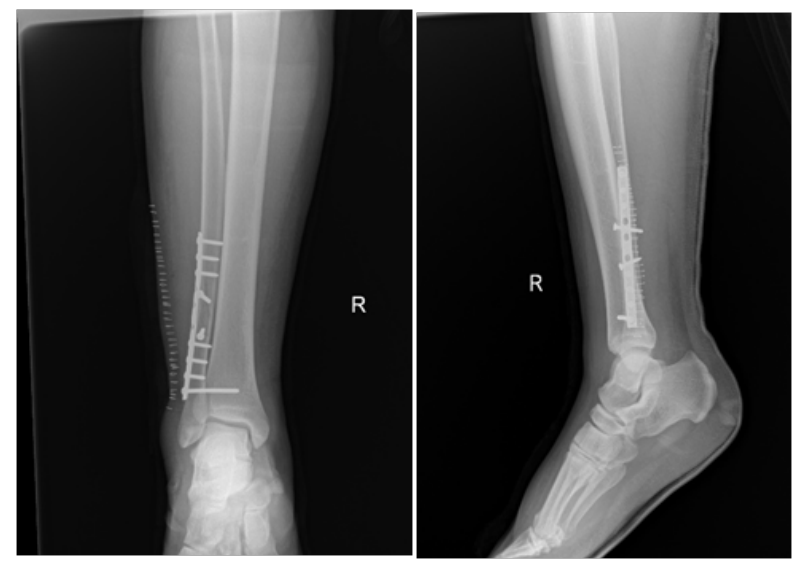

Figure $2 \mathrm{AP}$ and lateral $\mathrm{X}$-rays of right ankle showing after fixation.

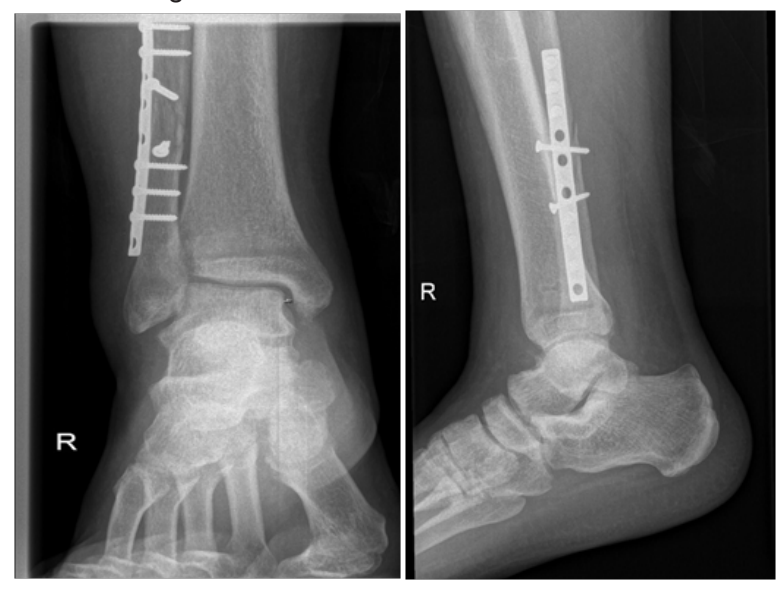

Figure 3 Synedsmotic screw removal and noticed big toe deformity.

\section{Treatment}

Patient was operated as a lateral malleolus fracture by plate along with syndesmotic screw.

\section{Outcome and follow-up}

After 6 weeks patient noticed big toe flexion contracture (Figure

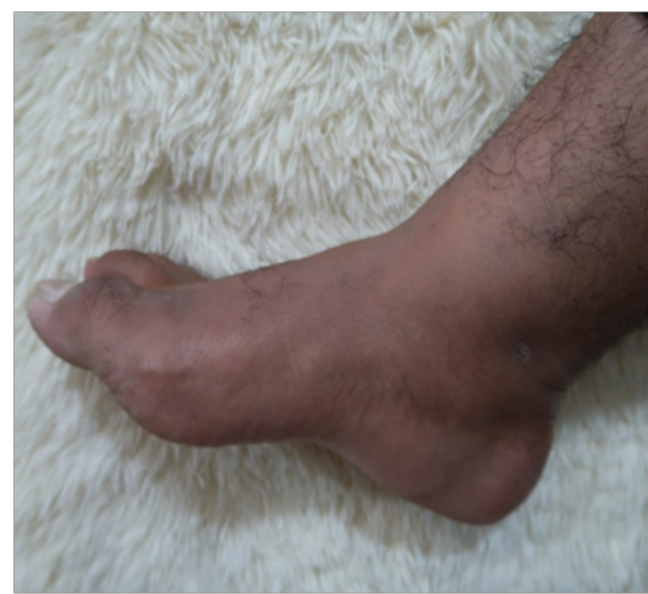

4) and started on splinting and stretching as treatment. At 8 weeks syndesmotic screw removed and he continued on conservative treatment for 1 year with no improvement of deformity. Then he underwent surgery for implant removal and midfoot FHL lengthening (Figure 5). Patient was followed for 2 month after surgery with full correction of the deformity.

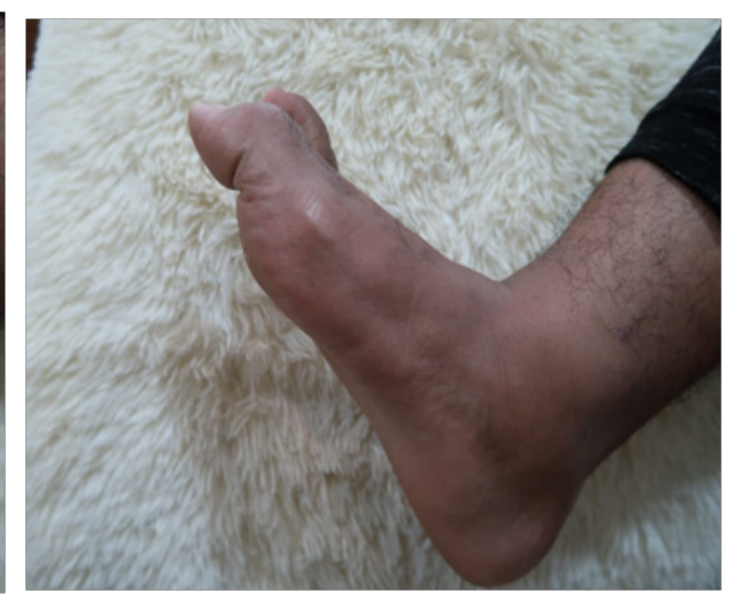

Figure 4 After 6 weeks patient noticed big toe flexion contracture. 


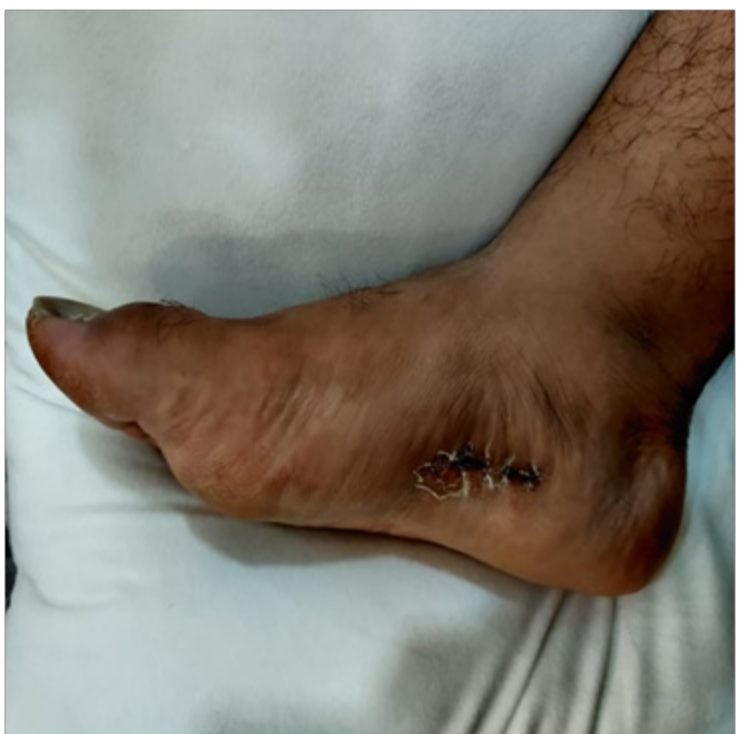

Figure 5 Showing implant removal and midfoot FHL lengthening.

\section{Discussion}

Flexor hallucis longus (FHL) muscle is one of the deep compartment of the leg. Its origin is from the body of the distal half of the fibula. ${ }^{4,5}$ The tendinous part begins just above the posterior aspect of medial malleolus and is located posterolateral to the posterior tibialis and the flexor digitorum longus tendons. The checkrein deformity is a dynamic flexion deformity of the big toe due to tethering of the tendon of FHL and may occurs after fracture of the lower limb. Flexion of interphalangeal joint of the big toe and sometimes the other toes becomes more visible when the ankle is passively dorsiflexed. On plantar flexion, the deformity is corrected completely. It was suggested that it results from scarring or callus formation from fracture healing. Surgical treatment includes either release of adhesion or lengthening of the FHL tendon. The lengthening of FHL is either done at fracture side or at midfoot. Lengthening of the FHL tendon at the midfoot is recommended, as it has a lower risk of adhesion and less rate of recurrence. ${ }^{6}$ At the midfoot, separation of FDL tendon attached to FHL tendon is required then Z-plasty lengthening of the FHL tendon. This is necessary to allow correction of deformity in the second toe with lengthening of the FHL tendon alone. ${ }^{7}$ Lengthening of FHL around the medial malleolar has a high rate of adhesion and recurrence, it could be due to musculotendinous junction of the FHL tendon proximity to the injury site. In our patient, lengthening of the FHL tendon alone was performed at the tarsal tunnel after releasing the FDL tendon.

\section{Learning points/take home messages}

FHL tethering after lower limb fracture mostly will not respond to conservative management. Midfoot lengthening is preferred over lengthening at the musculotendinous junction at a fracture site.

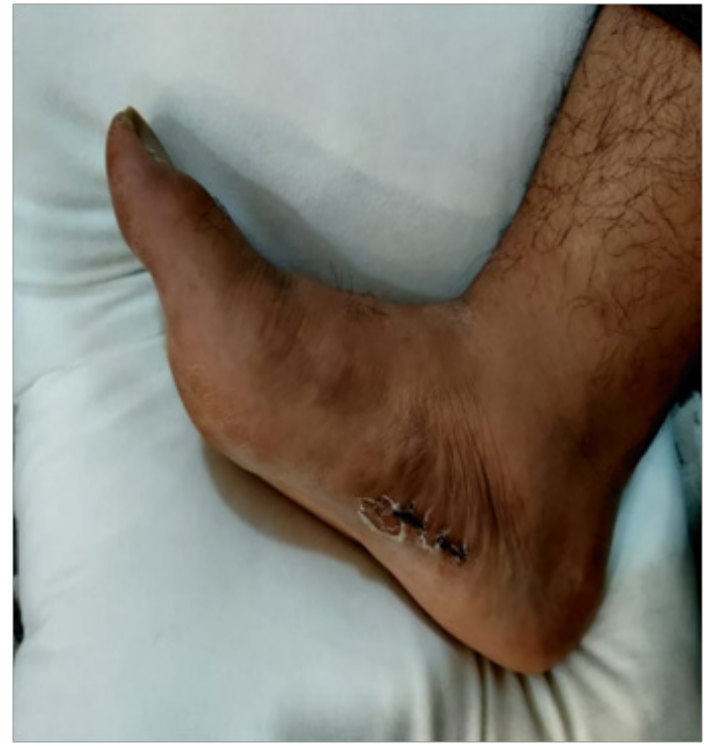

\section{Acknowledgments}

None.

\section{Conflicts of interest}

The authors declare there are no conflicts of interest.

\section{References}

1. Lui TH. Endoscopic adhesiolysis of the flexor hallucis longus muscle. Arthrosc Tech. 2017;6(2):e325-e329.

2. Carr JB. Complications of calcaneus fractures entrapment of the flexor hallucis longus: report of two cases. J Orthop Trauma. 1990;4(2):166168.

3. Feeney MS, Williams RL, Stephens MM. Selective lengthening of the proximal flexor tendon in the management of acquired claw toes. $J$ Bone Joint Surg [Br]. 2001;83(3):335-338.

4. Lo LD, Schweitzer ME, Fan JK, et al. MR imaging findings of entrapment of the flexor halluces longus tendon. AJR Am J Roentgenol. 2001;176(5):1145-1148.

5. Sassu P, Acland RD, Salgado CJ, et al. Anatomy and vascularization of the flexor hallucis longus muscle and its implication in free fibula flap transfer: an anatomical study. Ann Plast Surg. 2010;64(2):233-237.

6. Lee HS, Kim JS, Park SS, et al. Treatment of checkrein deformity of the hallux. J Bone Joint Surg Br. 2008;90(8):1055-1058.

7. Lee JH, Kim YJ, Baek JH, et al. Z-plasty of the flexor hallucis longus tendon at tarsal tunnel for checkrein deformity. J Orthop Surg (Hong Kong). 2016;24(3):354-357. 